\title{
The role of SPARC in extracellular matrix assembly
}

\author{
Amy D. Bradshaw
}

Received: 29 May 2009 / Accepted: 20 August 2009/Published online: 2 October 2009

(C) The Author(s) 2009. This article is published with open access at Springerlink.com

\begin{abstract}
SPARC is a collagen-binding matricellular protein. Expression of SPARC in adult tissues is frequently associated with excessive deposition of collagen and SPARC-null mice fail to generate a robust fibrotic response to a variety of stimuli. This review summarizes recent advancements in the characterization of the binding of SPARC to collagens and describes the results of studies that implicate a function for SPARC in the regulation of the assembly of basal lamina and fibrillar collagen in the ECM. Potential cellular mechanisms that underlie SPARC activity in ECM deposition are also explored.
\end{abstract}

Keywords BM-40 · Collagen · Fibrillogenesis ·

Osteonectin $\cdot$ Review

\section{Introduction}

Secreted Protein Acidic and Rich in Cysteine (SPARC, osteonectin, BM-40) is a highly conserved matricellular protein that is expressed in C. elegans, Drosophila, honey bee, brine shrimp, zebra fish, chicken, and mammals (Bradshaw and Sage 2001; Tanaka et al. 2001; Kucharski and Maleszka 2002; Rotllant et al. 2008). Worms and

\section{A. D. Bradshaw}

Division of Cardiology, Department of Medicine,

Medical University of South Carolina and the Ralph H. Johnson

Veteran's Administration,

Charleston, SC, USA

A. D. Bradshaw $(\bowtie)$

Gazes Cardiac Research Institute,

Medical University of South Carolina,

114 Doughty St.,

Charleston, SC 29425, USA

e-mail: bradshad@musc.edu flies, each with one gene encoding SPARC, do not survive abrogation of SPARC expression (Fitzgerald and Schwarzbauer 1998; Martinek et al. 2008). Ectopic expression and inhibition of SPARC expression in amphibians leads to developmental arrest (Purcell et al. 1993; Damjanovski et al. 1997). SPARC-null mice however, are viable and reproduce perhaps due to compensation from other SPARC family members such as hevin/SC1 and SMOC-2 (Sullivan and Sage 2004; Liu et al. 2008). Although a number of different activities are associated with SPARC, including processes regulating proliferation, growth factor activity, matrix metalloproteinase (MMP) activity, and migration - this review will focus on the interaction of SPARC with collagens and mechanism(s) by which SPARC might influence the assembly and deposition of the ECM (Brekken and Sage 2001).

\section{SPARC binding to collagens}

SPARC consists of 3 modular domains (Brekken and Sage 2001). The N-terminal region contains a low-affinity, highcapacity $\mathrm{Ca}^{2+}$-binding domain. The central portion of the protein includes a region with homology to follistatin, whereas the C-terminal, third domain contains two highaffinity $\mathrm{Ca}^{2+}$-binding EF hands. The domain of SPARC that binds collagens and cell surfaces is localized to the third domain. (Hohenester et al. 1997).

Although SPARC is reported to bind a number of ECM proteins, SPARC binding to collagens is the best characterized of these interactions. The binding of SPARC to collagens is $\mathrm{Ca}^{2+}$-dependent and requires non-denatured, triple helical collagen (Sasaki et al. 1998). The affinity of SPARC for collagen IV, the most 
abundant collagen in the basal lamina, exhibits a $\mathrm{K}_{\mathrm{d}}$ of $\sim 1-2 \mu \mathrm{m}$ (Sasaki et al. 1998). SPARC also binds to fibrillar collagens I, II, III, and V with a similar, or in some cases, a slightly reduced affinity (Sasaki et al. 1998). Cleavage of SPARC by MMPs at amino acid L197, or by an unidentified protease in tissues at residue L198 in helix $\alpha \mathrm{C}$ of the collagen-binding region, results in a ten-fold increase in the affinity of SPARC for collagens I, II, III, IV and V (Sasaki et al. 1997). As helix $\alpha \mathrm{C}$ partially masks the collagen-binding site in helix $\alpha \mathrm{A}$, protease cleavage is predicted to relieve this masking effect thereby increasing collagen binding. A recombinant form of SPARC (rSPARC), with aa 196-203 deleted mimics the effects of protease digestion and generates an "activated" form of recombinant SPARC (SPARC $\Delta \mathrm{I}, \alpha \mathrm{C}$; SPARC FS-EC $\Delta \alpha \mathrm{C}$ ) which engages collagen with a 10 -fold higher affinity than full-length rSPARC (Sasaki et al. 1997). Tissue-specific differential glycosylation of SPARC also influences SPARC binding to collagen. For example, the SPARC that is expressed in bone is modified with highmannose structures and exhibits a higher affinity for collagen I than SPARC from platelets that does not have the high-mannose modification (Kaufmann et al. 2004).

The SPARC-binding site on collagen I is described in two separate studies. Wang et al. (2005) identified SPARC binding sites on collagen I with atomic force microscopy using rSPARC (generated in insect cells) and rat tail collagen I or recombinant procollagen I. Similar binding to procollagen I and collagen extracted from rat tail (with propeptides removed) was demonstrated. Although evidence of SPARC binding to collagen I appeared all along the protein, two preferred sites of interaction with SPARC, a primary and a secondary site, were reported. The primary site localized to amino acid residues $\sim 650-800$ on collagen I $\sim 87.5-125 \mathrm{~nm}$ from the C-terminus of procollagen I whereas a secondary site mapped nearer the N-terminus, $\sim 237.5-262.5 \mathrm{~nm}$ from the C-terminus, corresponding to aa 100-200. The primary site at aa 650 800 overlaps a previously reported $\alpha_{1} \beta_{1}$ integrin recognition site, as well as binding sites for cartilage oligomeric protein (COMP/thrombospondin 5) and fibronectin, whereas the secondary site overlaps a potential $\alpha_{2} \beta_{1}$ integrin interaction site (Wang et al. 2005).

In a second study, Giudici et al. (2008), assessed SPARC collagen interactions by rotary shadowing, followed by electron microscopy. Using rSPARC made in mammalian cells with the helix $\alpha \mathrm{C}$ deleted to generate "activated" SPARC $\Delta \mathrm{I}, \alpha \mathrm{C}$ and human procollagen produced in vitro by skin fibroblasts, these authors identified a major SPARC binding site $\sim 180 \mathrm{~nm}$ from the C-terminus of collagen I. Likewise, a nearly identical region in fibrillar collagens II and III was shown as the most favored for interaction of SPARC in these studies.
Lesser sites of SPARC binding, in order of preference, were located at $\sim 80 \mathrm{~nm}$ and $\sim 240 \mathrm{~nm}$ from the C-terminus. Guidici et al. (2008) obtained a synthetic triple-helical peptide representing homotrimeric collagen III aa residues $397-423$ in the major binding site $(\sim 180 \mathrm{~nm}$ from the C-terminus) and found that SPARC binds this peptide with an affinity similar to that of full-length procollagen III. The analogous domain of collagen I is known to contain binding sites for von Willebrand factor (vWF) and Discoidin Domain Receptor (DDR) 2.

The primary disagreement between the two SPARC collagen I binding studies is that Wang et al. did not find a primary interaction site at the $\sim 180 \mathrm{~nm}$ region, containing the previously characterized vWF/DDR 2 binding domain. Both studies identified the $\sim 80 \mathrm{~nm}$ site and the lesser site $\sim 240 \mathrm{~nm}$ from the C-terminus as SPARC-binding regions. Whether differences in the source of SPARC (rSPARC from insect cells versus rSPARC $\Delta \mathrm{I}, \alpha \mathrm{C}$ from mammalian cells) underlie these results is currently not known. The source of human procollagen, versus recombinant procollagen, is perhaps another factor that influences the location of binding sites. Nonetheless, the identification of SPARC-binding sites on fibrillar collagens suggests that SPARC can influence the interaction of collagen with cell-surface collagen receptors of the integrin and/or DDR family, as well as sites of collagen interaction with other ECM components including fibronectin and/or collagen-binding proteoglycans.

A recent study by Hohenester et al. (2008) reports the crystal structure of activated rSPARC FS-EC $\Delta \alpha \mathrm{C}$ bound to a 33-residue peptide from collagen III representing aa residues $564-590$, the major binding site identified by Guidici et al. $\sim 180 \mathrm{~nm}$ from the C-terminus. SPARC bound the GVMGFO motif and buried a total of $720 \mathrm{~A}^{2}$ of solvent-accessible collagen surface. Engagement of collagen by SPARC is predicted to alter substantially the conformation of SPARC resulting in a deep pocket that accommodates the phenylalanine on the trailing collagen chain (Hohenester et al. 2008). In contrast, the triple helix of the collagen molecule does not undergo significant changes upon SPARC binding. Based on data from the crystal structure, the authors devised a sequence rule that predicts SPARC interaction sites elsewhere in the collagen molecule (Hohenester et al. 2008). A second site is predicted at the GATGFO sequence which is located at $\sim 80 \mathrm{~nm}$ from the C-terminus at residue $\sim 700$, as shown previously by atomic force microscopy and by rotary shadowing (Wang et al. 2005; Giudici et al. 2008). Using the same sequence rule, Hohenester et al. (2008) predict four SPARC-binding sites in collagen IV, $[\alpha 1 \text { (IV) }]_{2} \alpha 2$ (IV). Of these, one site at position aa 480 in COL4A1 is in close proximity to a previously identified integrin $\alpha_{1} \beta_{1}$ binding site (Kern et al. 1993). 


\section{SPARC and collagen ECM assembly}

Fibrillar collagens

The fact that SPARC binds to collagens suggests that SPARC might influence the assembly of collagen in the ECM. In fact, the absence of SPARC in mice results in significant differences in collagen fibril morphology, as well as substantial decreases in adult tissue concentrations of collagen. For example, the skin of SPARC-null mice has $\sim$ half the amount of collagen, as measured by hydroxyproline analysis, in comparison with wild-type skin (Bradshaw et al. 2003b). Similar decreases in interstitial collagen are apparent in heart and in fat depots of SPARCnull mice (Bradshaw et al. 2003a; Bradshaw et al. 2009). Collagen fibrils formed in the dermis in the absence of SPARC are smaller and more uniform in diameter than those of wild-type animals. Decreases in the average diameter of collagen fibrils in SPARC-null skin are significant at 1 month of age and become more substantial at 6 months of age (Rentz et al. 2007). At 1 month, the frequency of collagen fibrils with diameters of $60-70 \mathrm{~nm}$ is significantly elevated in the absence of SPARC (Rentz et al. 2007). This size of collagen fibril is proposed to be an intermediate in collagen fibril assembly that serves as a substrate for fibril: fibril aggregation to generate larger collagen fibrils (Ezura et al. 2000). SPARC might serve to augment collagen fibril fusion directly, and therefore collagen fibrils accumulate at a size of $60-70 \mathrm{~nm}$ in diameter in its absence.

However, developmental patterns of SPARC expression together with immunohistochemistry do not support a direct function of SPARC in mediating fibril fusion. Robust SPARC expression is evident in post-natal day-1 skin, and drops significantly by 2 weeks of age with a further decrease at 1 month (Bradshaw et al. 2003b). Collagen fibrils from 2 weeks through 1 month of age continue to undergo active fibril fusion. In addition, detection of SPARC in association with extracellular structures, such as collagen fibers, by immunohistochemistry, is infrequent in skin samples from animals older than post-natal day 1 (Bradshaw et al. 2003b).

Perhaps, rather than facilitating collagen fibril fusion directly, the activity of SPARC may be required to generate collagen fibrils with the capacity to fuse to form larger fibrils. Guidici et al. (2008) report that rSPARC inhibits collagen fibrillogenesis assays in vitro. rSPARC was shown to increase the length of the lag phase of fibrillogenesis, depending on the molar excess of SPARC over collagen used in the assay. The lag phase that occurs in vitro during collagen fibrillogenesis is thought to represent a nucleation step that is required to initiate collagen fibril formation, and that is followed by a fiber growth phase. The addition of equimolar amounts of SPARC $\Delta \mathrm{I}, \alpha \mathrm{C}$ to collagen inhibits collagen fibrillogenesis completely over the time of the assay. Addition of either rSPARC or SPARC $\Delta \mathrm{I}, \alpha \mathrm{C}$ following the lag phase, coincident with the middle of the growth phase, has no effect on fibrillogenesis in this assay (Giudici et al. 2008). Hence, the capacity of SPARC to influence fibrillogenesis is implicated during early steps in collagen fibril formation.

Basal lamina

Evidence that SPARC also influences basal lamina assembly, perhaps through an interaction with collagen IV, is supported by studies both in invertebrates and in vertebrates. SPARC labeled with green fluorescent protein localizes to basal lamina structures in C. elegans (Fitzgerald and Schwarzbauer 1998). Abrogation of SPARC expression in flies results in a lack of collagen IV in the basal lamina of certain structures in the embryo, notably in the ventral nerve chord, and results in embryonic lethality (Martinek et al. 2008). SPARC mutant flies display defects similar to those of collagen IV mutants suggesting an inter-dependency of these two proteins in embryonic basal lamina formation in flies (Martinek et al. 2008).

The phenotype of premature cataractogenesis in SPARC-null mice indicates that SPARC also contributes to basal lamina assembly in vertebrates (Gilmour et al. 1998). Immunohistochemistry of the basal lamina produced by lens epithelial cells shows that laminin and collagen IV are disorganized in the lens capsule of SPARC-null animals (Yan et al. 2002). Interestingly, whereas the cell membrane of wild-type epithelial cells forms a tight, linear surface adjacent to the basal lamina, SPARC-null epithelial cells appear convoluted, with abundant membrane protrusions extending into the basal lamina (Yan et al. 2002). Immunohistochemistry reveals that these cellular protrusions are $\beta_{1}$ integrin-positive. Perhaps SPARC bound to collagen IV diminishes interaction with cell-surface receptors, such as $\alpha_{1} \beta_{1}$, so that, in SPARC-null mice, increased receptor engagement tethers collagen IV to cell surfaces interfering with appropriate basal lamina assembly. The collagen IV network in the lens basement membrane is less stabilized by non-covalent and covalent forces in comparison to other basement membranes and therefore might prove more susceptible to alterations in structural integrity in the absence of SPARC (Vanacore et al. 2004). Results generated from SPARC-null lens epithelial cells also reveal an activity of SPARC in the regulation of laminin deposition as its absence results in an increase in amounts of laminin in the lens capsule and an increase in laminin deposited by SPARC-null versus wild-type lens epithelial cells in vitro (Yan et al. 2005). A recent report from Greiling et al. (2009) revealed a disruption in proper circulation of fluids and ions in 
SPARC-null lens that suggests the cellular basis of cataract formation in the absence of SPARC is likely influenced by multiple factors.

\section{Cellular mechanisms of SPARC in ECM assembly}

Regulation of collagen binding to cell surface receptors

SPARC purified from media conditioned by parietal yolk sac (PYS) cells was the first form of SPARC shown to induce rounding of endothelial cells in culture, and this together with subsequent studies have led to the characterization of SPARC as a counter-adhesive protein (Sage et al. 1989). Exogenous SPARC rounds cells but usually does not lead to cell lifting and subsequent cell death by anoikis, i.e. apoptosis induced by cell detachment. Removal of exogenous SPARC reverses rounding and cells reattach without significant cell death. The counter-adhesive activity of SPARC is consistent with an activity of SPARC that interferes with cell-ECM interactions. Along similar lines, the analysis of collagen interaction with primary dermal fibroblasts reveal an increase in procollagen, intermediates of procollagen processing, namely $\mathrm{pC}$ collagen $\mathrm{I}, \mathrm{pN}$ collagen I, and processed collagen I, in SPARC-null versus wild-type cells (Rentz et al. 2007). Given the binding sites of SPARC to collagen, one might predict that SPARC bound to collagen diminishes cell-surface receptor engagement and thus reduces cell adhesion, whereas collagen synthesized in the absence of SPARC readily associates with cell receptors. Alternatively, SPARC has also recently been shown to affect integrin-linked kinase (ILK) activity that is predicted to influence integrin ligation independent of SPARC: collagen interactions (Barker et al. 2005; Nie and Sage 2009). The role of SPARC in the modulation of ILK function and downstream pathways is covered in more detail in the review of SPARC in adipocyte biology by Nie and Sage in this series.

If SPARC functions to limit collagen engagement with cell-surface receptors, then could the phenotypic alterations in ECM assembly, noted in SPARC-null mice, result from an increased interaction of collagen with its receptors? The primary SPARC-binding site on collagen described by Guidici et al. (2008) and further assessed in Hohenester et al. (2008), is coincident with the DDR2-binding site on collagen I. DDR2 was shown to inhibit collagen fibrillogenesis in cultured cells (Mihai et al. 2006; Vogel et al. 2006). Hence, if the absence of SPARC promotes engagement of collagen by DDR2, then collagen fibril formation might be reduced and/or altered. Reductions in collagen concentration and compromised collagen fibril assembly in SPARC-null mice might result from an increase in DDR2 to collagen interactions.
The ligation of collagen-binding integrin receptors, namely $\alpha_{1} \beta_{1}, \alpha_{2} \beta_{1}$, and $\alpha_{11} \beta_{1}$, may also be diminished by SPARC binding to collagen (White et al. 2004). In contrast to DDR2, integrin collagen receptors are thought to contribute to the formation and/or stability of collagen fibrils. In vitro, fibronectin assembly precedes and is required for robust collagen fibril assembly (Velling et al. 2002). Collagen-binding integrin receptors enhance fibronectin-dependent collagen fibrillogenesis in culture. For example, in vascular smooth muscle cells, a decrease in collagen fibrils is observed on cells treated with functionblocking anti- $\alpha_{2} \beta_{1}$ IgG versus control IgG (Li et al. 2003). Similarly, in mouse embryonic fibroblasts, fibronectin is required for collagen fibrillogenesis while $\alpha_{11} \beta_{1}$, and $\alpha_{2} \beta_{1}$ are shown to enhance this process (Velling et al. 2002). Therefore, an increase in integrin engagement might be predicted to facilitate collagen fibril assembly and increase collagen concentrations in tissues, a result not in line with the ECM phenotype of SPARC-null tissues.

In addition to its functional contributions to ECM assembly, integrin $\alpha_{2} \beta_{1}$ is also known to mediate collagen phagocytosis (Lee et al. 1996). In this case, the lack of SPARC expression is anticipated to increase collagen turnover at the cell surface through elevated uptake of collagen by $\alpha_{2} \beta_{1}$ engagement. A plausible scenario might be one in which SPARC, a protein that supports collagen deposition, interferes with receptor interactions on collagen that inhibit fibril formation and/or promote collagen turnover (Fig. 1).

\section{Regulation of processing of procollagen propeptides}

Whether SPARC acts exclusively in the extracellular space to modulate collagen interactions with cell-surface receptors or acts intracellularly in collagen trafficking and posttranslation modification remains to be elucidated. SPARC might influence collagen I production and deposition at a number of different steps. A series of post-translational modifications of collagen I occur in the endoplasmic reticulum, the cis-Golgi, and possibly in trans-Golgi compartments, that produce hydroxylated amino acids, sugar modifications with carbohydrates, trimeric chain assembly, and finally secretion of correctly assembled procollagen I (Prockop and Kivirikko 1995). SPARC is also present in the secretory pathway raising the possibility that intracellular interaction of SPARC with collagen I might influence collagen I modifications and assembly. The hypothesis that SPARC serves as an intracellular collagen chaperone that is important in the latter stages of collagen secretion was put forth by Martinek et al. (2007) based primarily on results from experiments with Drosophila and collagen IV. Along these lines, chaperone folding activity is exhibited by SPARC in assays performed in vitro (Emerson et al. 2006). 


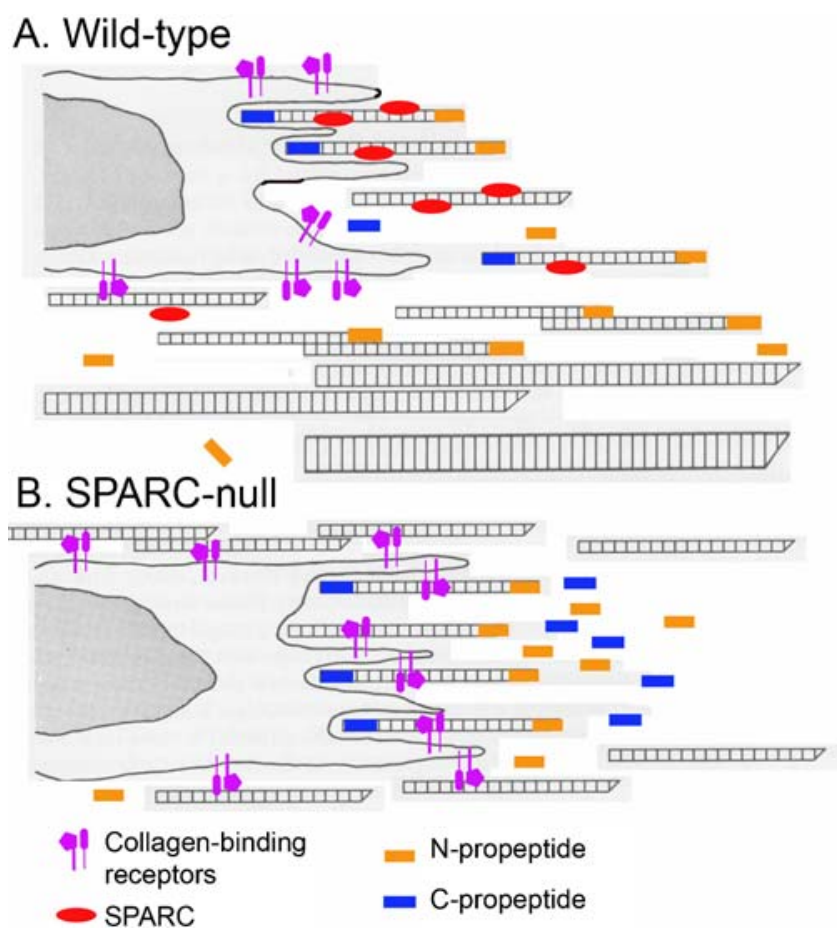

Fig. 1 SPARC activity in modulating collagen cell interaction and procollagen processing. In a, procollagen fibrils are bound by SPARC, which diminishes collagen engagement by cell-surface receptors. In the absence of SPARC $\mathbf{b}$, procollagen interacts with receptors to a greater degree and is tethered to cell surfaces. Propeptide cleavage is enhanced in the pericellular milieu. SPARC-null fibrils fail to aggregate as efficiently as fibrils on wt cells. One SPARC molecule is shown here binding to one collagen molecule for simplicity. Three sites are known to preferentially bind SPARC on the collagen molecule albeit with varying degrees of efficiency. From (Rentz et al. 2007) with permission

However, at least in dermal fibroblasts, intracellular accumulation of procollagen I is not evident in SPARC-null fibroblasts as would be predicted in the event that SPARC were critical for post-translational modification and/or sorting of procollagens (Rentz et al. 2007). Once secreted, procollagen I is converted to mature collagen I by N-propeptide proteinases, specifically ADAMTS-2 (A Disintegrin And Metalloproteinase with ThromboSpondin motifs), that excises the N-terminal propeptide to generate $\mathrm{pC}$ collagen $\mathrm{I}$, and by the C-propeptide proteinase, Bone Morphogenic Protein (BMP)-1, that excises the C-propeptide to produce $\mathrm{pN}$ collagen I (Porter et al. 2005; Hopkins et al. 2007). The collagen on primary murine dermal fibroblast cell surfaces is therefore comprised of four different forms: procollagen I, $\mathrm{pC}$ collagen $\mathrm{I}, \mathrm{pN}$ collagen $\mathrm{I}$, and collagen I. In the absence of SPARC, in addition to an increase in total amounts of cell-associated collagen I, processed collagen I (with both propeptides removed) represents a greater proportion of total collagen than in wild-type cells (Rentz et al. 2007). Hence, SPARC appears to serve a function in the regulation of procolla- gen processing that is generally considered to occur in the extracellular space. Although in embryonic tendon, some intracellular processing of propeptides is observed indicating that, at least in some cell types, procollagen processing can occur in secretory vesicles prior to secretion (Canty and Kadler 2005). The mechanism by which SPARC modulates procollagen processing, whether intra or extracellular, is actively under investigation.

In some cases, propeptides are retained following incorporation into fibrils (Fleischmajer et al. 1988). During development in the skin of chicks, antibodies against the Npropeptide of collagen I were immuno-localized exclusively to the surface of collagen fibrils $20-40 \mathrm{~nm}$ in diameter (Fleischmajer et al. 1983). Hence, processing of the propeptides of collagen I represents a possible mechanism in the regulation collagen fibrillogenesis (Holmes et al. 1996). Retention of the propeptides of collagen I (and proteins bound to propeptides) following incorporation into fibrils, for example, could present a steric hindrance to fibril aggregation and thus limit fibril expansion.

Alternatively, some evidence suggests that the N-propeptide acts to enhance collagen I fibril aggregation (Watson et al. 1998). In this case, premature processing of the N-propeptide might limit fibril expansion. In vitro, the absence of SPARC is associated with greater procollagen processing, as judged by propeptide cleavage. In the event that the absence of SPARC in vivo results in a similar increase, perhaps the premature removal of the N-propeptide generates collagen fibrils without $\mathrm{N}$-propeptides that are less efficient in fibril fusion. Given the result that SPARC inhibits collagen fibrillogenesis in vitro, specifically during the lag phase of initiation, one possibility is that SPARC binds nascent monomeric collagen I (and/or procollagen I) and delays its incorporation into nascent fibrils until events such as propeptide processing and/or engagement of proteoglycans (including fibromodulin, lumican, decorin, etc.) occur in a precise spatial and temporal sequence in the pericellular space. In the absence of SPARC expression then, the precise regulation of procollagen maturation to collagen is altered and the efficiency of collagen deposition to the insoluble ECM is compromised.

\section{SPARC as an anti-fibrotic target or promoter of ECM assembly}

SPARC expression is consistently found coincident with expression of fibrillar collagens and is therefore frequently elevated in adult tissues undergoing wound repair or in tissues with fibrotic disease. Mouse models characterized by renal, cardiac, lung, and dermal fibrosis performed in SPARC-null mice demonstrate reduced collagen deposition versus that in wild-type mice (Strandjord et al. 1999; 
Bradshaw et al. 2002; Socha et al. 2007; Bradshaw et al. 2009). Given the significant effects of the loss of SPARC expression on collagen levels in SPARC-null mice, SPARC represents an attractive target for reducing collagen deposition in fibrosis. Scleroderma is a disease characterized by fibrotic deposition of collagen. Targeting SPARC expression by siRNA in fibroblasts from individuals with scleroderma reduces collagen I expression in vitro (Zhou et al. 2006). Furthermore, in a rat model of liver fibrosis, Camino et al. (2008) demonstrated that anti-sense RNA against SPARC, delivered by adenovirus, reduces collagen deposition as measured by histological and by hydroxyproline analysis. These studies suggest that inhibition of SPARC is sufficient to decrease collagen accumulation in tissues and further validate the development of tools that target SPARC activity to alleviate fibrosis in a variety of tissues.

As a reduction in SPARC expression diminishes collagen accumulation in the ECM, an increase in SPARC expression is anticipated to enhance collagen deposition. In heart, myocardial infarction (MI) is associated with rapid synthesis of a collagenous scar to reduce the risk of cardiac rupture. SPARCnull mice are compromised in myocardial collagen formation and exhibit a greater mortality due to cardiac rupture following MI in comparison with wild-type mice (Bradshaw et al. 2009; Schellings et al. 2009). The production of SPARC, delivered by adenoviral expression in SPARC-null animals, improved collagen fiber assembly and reduced the mortality of SPARC-null animals with infarctions to that of wild-type mice (Schellings et al. 2009). Importantly, overexpression of SPARC by adenoviral delivery in wild-type animals subjected to infarction further enhanced collagen assembly in these mice and improved cardiac function (Schellings et al. 2009). Similar results from experiments using injected glioma cells demonstrated increased collagenous ECM production in vivo, that was associated with cells that over-expressed SPARC, in comparison with the use of control cells (Yunker et al. 2008). Taken together, these studies provide evidence that increased expression of SPARC stimulates increased collagen deposition and assembly into the ECM.

The evidence that SPARC influences TGF- $\beta$ activity has been reported in several different cell types. In epithelial cells and in mesangial cells, SPARC was shown to enhance TGF- $\beta 1$ activity through an as yet undefined mechanism (Schiemann et al. 2003; Francki et al. 2004). In addition, resident cells of the infarct scar in wild-type mouse hearts with over-expression of SPARC demonstrated increased levels of activated Smad2, a down-stream element in the TGF- $\beta 1$ signaling cascade (Schellings et al. 2009). Hence, there is concern that long term exposure to elevated levels of SPARC in a therapeutic setting might give rise to unexpected deleterious outcomes associated with increased TGF- $\beta$ activity. Further characterization of the mechanism (s) by which SPARC modulates TGF- $\beta 1$ signaling will provide insight in this regard.

\section{Conclusions}

In this review, a summary of results that support a function for SPARC as a critical factor in the formation of a collagenous ECM has been presented. In particular, SPARC is shown to play a prominent role in the deposition of fibrotic collagen in adult tissues. As such, analyses to elucidate the functional basis of SPARC activity in the regulation of collagen assembly have the potential to provide new insight into synthesis of the ECM and to suggest ways to control the formation of a pathological ECM. Conversely, in instances in which collagen deposition is required, for example in the synthesis of bioengineered tissues, an increased expression of SPARC can be expected to facilitate and enhance ECM assembly.

SPARC can influence the interaction of collagen with the cell surface possibly through modulation of receptor binding. In addition, purified SPARC inhibits collagen fibrillogenesis in a cell autonomous manner during early stages of fibril initiation in vitro. Thus SPARC has at least two roles in collagen fibril assembly-one that modulates interaction of collagen with the cell surface and another that regulates collagen incorporation to fibrils. Whether similar mechanisms involving the interaction of SPARC with collagen IV regulate basal lamina assembly is currently not known. Much remains to be learned from future studies that will hopefully discover the precise mechanisms that govern SPARC activity in ECM formation. These studies will no doubt bring novel and exciting ideas to the field of collagen and matricellular biology.

Acknowledgements ADB is supported by a Merit Award from the Veteran's Administration and by P20 2P20RR017696-07 from the NIDCR, NIH.

Open Access This article is distributed under the terms of the Creative Commons Attribution Noncommercial License which permits any noncommercial use, distribution, and reproduction in any medium, provided the original author(s) and source are credited.

\section{References}

Barker TH, Baneyx G, Cardo-Vila M, Workman GA, Weaver M, Menon PM, Dedhar S, Rempel SA, Arap W, Pasqualini R, Vogel V, Sage EH (2005) SPARC regulates extracellular matrix organization through its modulation of integrin-linked kinase activity. J Biol Chem 280:36483-36493

Bradshaw AD, Baicu CF, Rentz TJ, Van Laer AO, Boggs J, Lacy JM, Zile MR (2009) Pressure overload-induced alterations in fibrillar collagen content and myocardial diastolic function: role of 
secreted protein acidic and rich in cysteine (SPARC) in postsynthetic procollagen processing. Circulation 119:269-280

Bradshaw AD, Graves DC, Motamed K, Sage EH (2003a) SPARC-null mice exhibit increased adiposity without significant differences in overall body weight. Proc Natl Acad Sci U S A 100:6045-6050

Bradshaw AD, Puolakkainen P, Dasgupta J, Davidson JM, Wight TN, Sage EH (2003b) SPARC-null mice display abnormalities in the dermis characterized by decreased collagen fibril diameter and reduced tensile strength. J Invest Dermatol 120:949-955

Bradshaw AD, Reed MJ, Sage EH (2002) SPARC-null mice exhibit accelerated cutaneous wound closure. J Histochem Cytochem 50:1-10

Bradshaw AD, Sage EH (2001) SPARC, a matricellular protein that functions in cellular differentiation and tissue response to injury. J Clin Invest 107:1049-1054

Brekken RA, Sage EH (2001) SPARC, a matricellular protein: at the crossroads of cell-matrix communication. Matrix Biol 19:816827

Camino AM, Atorrasagasti C, Maccio D, Prada F, Salvatierra E, Rizzo M, Alaniz L, Aquino JB, Podhajcer OL, Silva M, Mazzolini G (2008) Adenovirus-mediated inhibition of SPARC attenuates liver fibrosis in rats. J Gene Med. 10:993-1004

Canty EG, Kadler KE (2005) Procollagen trafficking, processing and fibrillogenesis. J Cell Sci 118:1341-1353

Damjanovski S, Karp X, Funk S, Sage EH, Ringuette MJ (1997) Ectopic expression of SPARC in Xenopus embryos interferes with tissue morphogenesis: identification of a bioactive sequence in the C-terminal EF hand. J Histochem Cytochem 45:643-655

Emerson RO, Sage EH, Ghosh JG, Clark JI (2006) Chaperone-like activity revealed in the matricellular protein SPARC. J Cell Biochem 98:701-705

Ezura Y, Chakravarti S, Oldberg A, Chervoneva I, Birk DE (2000) Differential expression of lumican and fibromodulin regulate collagen fibrillogenesis in developing mouse tendons. J Cell Biol 151:779-788

Fitzgerald MC, Schwarzbauer JE (1998) Importance of the basement membrane protein SPARC for viability and fertility in Caenorhabditis elegans. Curr Biol 8:1285-1288

Fleischmajer R, Olsen BR, Timpl R, Perlish JS, Lovelace O (1983) Collagen fibril formation during embryogenesis. Proc Natl Acad Sci U S A 80:3354-3358

Fleischmajer R, Perlish JS, Timpl R, Olsen BR (1988) Procollagen intermediates during tendon fibrillogenesis. J Histochem Cytochem 36:1425-1432

Francki A, McClure TD, Brekken RA, Motamed K, Murri C, Wang T, Sage EH (2004) SPARC regulates TGF-beta1-dependent signaling in primary glomerular mesangial cells. J Cell Biochem 91:915-925

Gilmour DT, Lyon GJ, Carlton MB, Sanes JR, Cunningham JM, Anderson JR, Hogan BL, Evans MJ, Colledge WH (1998) Mice deficient for the secreted glycoprotein SPARC/osteonectin/BM40 develop normally but show severe age-onset cataract formation and disruption of the lens. Embo J 17:1860-1870

Giudici C, Raynal N, Wiedemann H, Cabral WA, Marini JC, Timpl R, Bachinger HP, Farndale RW, Sasaki T, Tenni R (2008) Mapping of SPARC/BM-40/osteonectin-binding sites on fibrillar collagens. J Biol Chem 283:19551-19560

Greiling TM, Stone B, Clark JI (2009) Absence of SPARC leads to impaired lens circulation. Exp Eye Res: Epub ahead of print

Hohenester E, Maurer P, Timpl R (1997) Crystal structure of a pair of follistatin-like and EF-hand calcium-binding domains in BM-40. Embo J 16:3778-3786

Hohenester E, Sasaki T, Giudici C, Farndale RW, Bachinger HP (2008) Structural basis of sequence-specific collagen recognition by SPARC. Proc Natl Acad Sci U S A 105:18273-18277

Holmes DF, Watson RB, Chapman JA, Kadler KE (1996) Enzymic control of collagen fibril shape. J Mol Biol 261:93-97
Hopkins DR, Keles S, Greenspan DS (2007) The bone morphogenetic protein 1/Tolloid-like metalloproteinases. Matrix Biol 26:508-523

Kaufmann B, Muller S, Hanisch FG, Hartmann U, Paulsson M, Maurer P, Zaucke F (2004) Structural variability of BM-40/ SPARC/osteonectin glycosylation: implications for collagen affinity. Glycobiology 14:609-619

Kern A, Eble J, Golbik R, Kuhn K (1993) Interaction of type IV collagen with the isolated integrins alpha 1 beta 1 and alpha 2 beta 1. Eur J Biochem 215:151-159

Kucharski R, Maleszka R (2002) Evaluation of differential gene expression during behavioral development in the honeybee using microarrays and northern blots. Genome Biol 3: RESEARCH0007

Lee W, Sodek J, McCulloch CA (1996) Role of integrins in regulation of collagen phagocytosis by human fibroblasts. J Cell Physiol 168:695-704

Li S, Van Den Diepstraten C, D'Souza SJ, Chan BM, Pickering JG (2003) Vascular smooth muscle cells orchestrate the assembly of type I collagen via alpha2beta1 integrin, RhoA, and fibronectin polymerization. Am J Pathol 163:1045-1056

Liu P, Lu J, Cardoso WV, Vaziri C (2008) The SPARC-related Factor SMOC-2 promotes growth factor-induced Cyclin D1 expression and DNA synthesis via integrin-linked Kinase. Mol Biol Cell 19:248-261

Martinek N, Shahab J, Saathoff M, Ringuette M (2008) Haemocytederived SPARC is required for collagen-IV-dependent stability of basal laminae in Drosophila embryos. J Cell Sci 121:16711680

Martinek N, Shahab J, Sodek J, Ringuette M (2007) Is SPARC an evolutionarily conserved collagen chaperone? J Dent Res 86:296-305

Mihai C, Iscru DF, Druhan LJ, Elton TS, Agarwal G (2006) Discoidin domain receptor 2 inhibits fibrillogenesis of collagen type 1 . J Mol Biol 361:864-876

Nie J, Sage EH (2009) SPARC inhibits adipogenesis by its enhancement of beta-catenin signaling. J Biol Chem 284:1279-1290

Porter S, Clark IM, Kevorkian L, Edwards DR (2005) The ADAMTS metalloproteinases. Biochem J 386:15-27

Prockop DJ, Kivirikko KI (1995) Collagens: molecular biology, diseases, and potentials for therapy. Annu Rev Biochem 64:403-434

Purcell L, Gruia-Gray J, Scanga S, Ringuette M (1993) Developmental anomalies of Xenopus embryos following microinjection of SPARC antibodies. J Exp Zool 265:153-164

Rentz TJ, Poobalarahi F, Bornstein P, Sage EH, Bradshaw AD (2007) SPARC regulates processing of procollagen I and collagen fibrillogenesis in dermal fibroblasts. J Biol Chem 282:22062-22071

Rotllant J, Liu D, Yan YL, Postlethwait JH, Westerfield M, Du SJ (2008) Sparc (Osteonectin) functions in morphogenesis of the pharyngeal skeleton and inner ear. Matrix Biol 27:561572

Sage H, Vernon RB, Funk SE, Everitt EA, Angello J (1989) SPARC, a secreted protein associated with cellular proliferation, inhibits cell spreading in vitro and exhibits $\mathrm{Ca}+2$-dependent binding to the extracellular matrix. J Cell Biol 109:341-356

Sasaki T, Gohring W, Mann K, Maurer P, Hohenester E, Knauper V, Murphy G, Timpl R (1997) Limited cleavage of extracellular matrix protein BM-40 by matrix metalloproteinases increases its affinity for collagens. J Biol Chem 272:9237-9243

Sasaki T, Hohenester E, Gohring W, Timpl R (1998) Crystal structure and mapping by site-directed mutagenesis of the collagen-binding epitope of an activated form of BM-40/SPARC/osteonectin. Embo J 17:1625-1634

Schellings MW, Vanhoutte D, Swinnen M, Cleutjens JP, Debets J, van Leeuwen RE, d'Hooge J, Van de Werf F, Carmeliet P, Pinto YM, Sage EH, Heymans S (2009) Absence of SPARC results in increased cardiac rupture and dysfunction after acute myocardial infarction. J Exp Med 206:113-123 
Schiemann BJ, Neil JR, Schiemann WP (2003) SPARC inhibits epithelial cell proliferation in part through stimulation of the transforming growth factor-beta-signaling system. Mol Biol Cell 14:3977-3988

Socha MJ, Manhiani M, Said N, Imig JD, Motamed K (2007) Secreted protein acidic and rich in cysteine deficiency ameliorates renal inflammation and fibrosis in angiotensin hypertension. Am J Pathol 171:1104-1112

Strandjord TP, Madtes DK, Weiss DJ, Sage EH (1999) Collagen accumulation is decreased in SPARC-null mice with bleomycininduced pulmonary fibrosis. Am J Physiol 277:L628-635

Sullivan MM, Sage EH (2004) Hevin/SC1, a matricellular glycoprotein and potential tumor-suppressor of the SPARC/BM-40/ Osteonectin family. Int J Biochem Cell Biol 36:991-996

Tanaka S, Nambu F, Nambu Z (2001) Isolation of a cDNA encoding a putative SPARC from the brine shrimp, Artemia franciscana. Gene 268:53-58

Vanacore RM, Shanmugasundararaj S, Friedman DB, Bondar O, Hudson BG, Sundaramoorthy M (2004) The alpha1.alpha2 network of collagen IV. Reinforced stabilization of the noncollagenous domain-1 by noncovalent forces and the absence of Met-Lys cross-links. J Biol Chem 279:44723-44730

Velling T, Risteli J, Wennerberg K, Mosher DF, Johansson S (2002) Polymerization of type I and III collagens is dependent on fibronectin and enhanced by integrins alpha 11 beta 1 and alpha 2beta 1. J Biol Chem 277:37377-37381
Vogel WF, Abdulhussein R, Ford CE (2006) Sensing extracellular matrix: an update on discoidin domain receptor function. Cell Signal 18:1108-1116

Wang H, Fertala A, Ratner BD, Sage EH, Jiang S (2005) Identifying the SPARC binding sites on collagen I and procollagen I by atomic force microscopy. Anal Chem 77:6765-6771

Watson RB, Holmes DF, Graham HK, Nusgens BV, Kadler KE (1998) Surface located procollagen N-propeptides on dermatosparactic collagen fibrils are not cleaved by procollagen N-proteinase and do not inhibit binding of decorin to the fibril surface. J Mol Biol 278:195-204

White DJ, Puranen S, Johnson MS, Heino J (2004) The collagen receptor subfamily of the integrins. Int J Biochem Cell Biol 36:1405-1410

Yan Q, Clark JI, Wight TN, Sage EH (2002) Alterations in the lens capsule contribute to cataractogenesis in SPARC-null mice. J Cell Sci 115:2747-2756

Yan Q, Perdue N, Blake D, Sage EH (2005) Absence of SPARC in murine lens epithelium leads to increased deposition of laminin-1 in lens capsule. Invest Ophthalmol Vis Sci 46:4652-4660

Yunker CK, Golembieski W, Lemke N, Schultz CR, Cazacu S, Brodie C, Rempel SA (2008) SPARC-induced increase in glioma matrix and decrease in vascularity are associated with reduced VEGF expression and secretion. Int J Cancer 122:2735-2743

Zhou X, Tan FK, Guo X, Arnett FC (2006) Attenuation of collagen production with small interfering RNA of SPARC in cultured fibroblasts from the skin of patients with scleroderma. Arthritis Rheum 54:2626-2631 